\title{
Transfer reaction studies in inverse kinematics with the magnetic spectrom- eter PRISMA
}

\author{
L.Corradi ${ }^{1, a}$ \\ ${ }^{1}$ INFN - Laboratori Nazionali di Legnaro - Viale dell'Universita' 2, 35020, Legnaro (Padova) - Italy
}

\begin{abstract}
.
The large solid angle magnetic spectrometers, in combination with large gamma arrays, allowed to perform reaction mechanism and nuclear structure studies in different regions of the nuclear chart, especially in the neutron-rich direction. By studying transfer of multiple pairs valuable information on nucleon-nucleon correlations can be derived, especially from measurements performed below the Coulomb barrier. There is growing interest in the study of the properties of the heavy binary partner, in the $\mathrm{Pb}$ and in the actinides regions, crucial also for astrophysics.
\end{abstract}

\section{Introduction}

By studying multinucleon transfer reactions at Coulomb barrier energies one can investigate nucleon-nucleon correlation in nuclei [1], the transition from the quasi-elastic to the deep-inelastic regime [2,3] and channel coupling effects in sub-barrier fusion reactions [4]. For more than one decade this mechanism is at a renewed focus of important experimental [5] and theoretical [6-8] advances. The advent of the last generation large solid angle magnetic spectrometers [9-12] coupled to large gamma arrays [13-15] allowed to perform gamma-particle coincidences, thus studying at the same time reaction mechanism and nuclear structure for nuclei produced via nucleon transfer or deep-inelastic reactions, especially in the neutron-rich region. Ongoing studies are of primary importance for reactions to be done with radioactive ion beams [16, 17] where multinucleon transfer has been shown to be a competitive tool for the study of neutron-rich nuclei, at least for certain mass regions. In the following, selected examples of recently studied reactions will be presented, with emphasis on experiments performed in inverse kinematics at sub-barrier energies and in the heavy mass regions [18].

\section{Reactions at sub-barrier energies}

At energies well below the Coulomb barrier, the interacting nuclei are only slightly influenced by the nuclear potential and $Q$ values are restricted to few $\mathrm{MeV}$ for the open transfer channels. These conditions diminish the complexity of coupled channel calculations and quantitative information may be extracted on the nucleon-nucleon correlations [19-21]. In literature many data on transfer reactions

\footnotetext{
a e-mail: corradi@lnl.infn.it
}

have been represented via the transfer probability $\left(P_{t r}\right)$, defined as the ratio of the transfer cross section to the corresponding Rutherford cross section, plotted as a function of the distance of closest approach $(D)$ but quite contradictory conclusion have been extracted, since data have been mostly taken at energies above the Coulomb barrier.

The coming into operation of large acceptance magnetic spectrometers made it possible to perform measurements on multinucleon transfer reactions with good ion identification also at very low bombarding energies [22]. With this experimental advance we are now in position to study the interplay between single and pair transfers far below the Coulomb barrier and thus to provide an answer on the origin of the enhancement reported for these reactions (see e.g. Ref. [23] and references therein). In very recently measured systems one successfully demonstrated the powerful method of using PRISMA for such studies, exploiting its unique performance in terms of both resolution and efficiency. Making use of inverse kinematics, target recoils have been detected in multinucleon transfer reactions for the systems ${ }^{96} \mathrm{Zr}+{ }^{40} \mathrm{Ca}[22]$ and ${ }^{116} \mathrm{Sn}+{ }^{60} \mathrm{Ni}$ [24]. In both cases the excitation functions at several bombarding energies have been obtained from the Coulomb barrier to $20-25 \%$ below, reaching about 15.5 fm of distance of closest approach.

Results of the measurement of the ${ }^{96} \mathrm{Zr}+{ }^{40} \mathrm{Ca}$ system (closed shell nuclei) are presented, together with the calculations, in Fig. 1 for $(+1 n)$ and $(+2 n)$ neutron transfer channels via transfer probabilities as a function of the distance of closest approach. Transfer yield could be detected at the level of $10^{-4}$ with respect to the elastic channel. To compute the inclusive one-neutron stripping cross section (full line) one calculated the transfer probability for a given single particle transition and one obtained the total transfer probability by summing over all possible transi- 


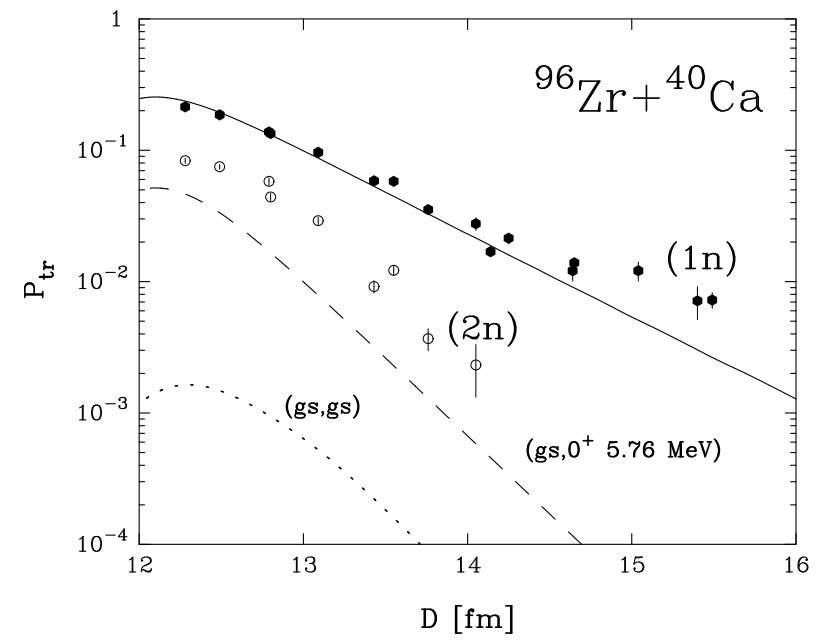

Figure 1. Points: Experimental transfer probabilities as a function of the distance of closest approach for $(+1 n)$ (full circles) and $(+2 n)$ (empty circles) for the ${ }^{96} \mathrm{Zr}+{ }^{40} \mathrm{Ca}$ system. Lines: Theoretical transfer probabilities for one and two particle transfer. The full line represents the inclusive transfer probability for one neutron transfer, the dotted line the ground to ground state transition for the two-neutron transfer and the dash line the transition to the first $0^{+}$excited state at $\sim 5.8 \mathrm{MeV}$ in ${ }^{42} \mathrm{Ca}$

tions that can be constructed from the single particle states in projectile and target (see Ref. [22] for details). One sees how calculations reproduce well the experimental slope as well as the absolute values of the transfer probabilities for the one neutron channel. For the two-particle transfer one followed closely the formalism of Ref. [21]. We here just mention that the ground state wave function for the ${ }^{94} \mathrm{Zr}$ is obtained from a BCS calculation by adopting a state independent pairing interaction, while for the description of ${ }^{42} \mathrm{Ca}$ one diagonalized the total Hamiltonian with a model space containing only two-particle configuration coupled to $0^{+}$(i.e. transfer of a $J=0^{+}$pair). In Fig. 1 with a dotted line is shown the calculated probability for the ground to ground state transition. Clearly, this transition does not contribute to the total transfer strength in agreement with what was experimentally observed in the $Q$-value spectra. The predicted transfer probability for the transition to the $0^{+}$state at $\sim 5.8 \mathrm{MeV}$ in ${ }^{42} \mathrm{Ca}[25]$ is shown with a dashed line. It is apparent that the contribution of this transition is much larger than the ground state one. One presently ascribes the enhancement factor of $\sim 3$ to the fact that the two-nucleon transfer reaction does not populate only $0^{+}$ states but it is much richer, so that more complicated twoparticle correlations have to be taken into account.

At variance with the ${ }^{96} \mathrm{Zr}+{ }^{40} \mathrm{Ca}$ system (closed shell), in the very recently studied ${ }^{116} \mathrm{Sn}+{ }^{60} \mathrm{Ni}$ system (super-fluid nuclei) [24], the ground to ground state $Q$ values for neutron transfers is close to zero, matching the optimum $Q$ value $(\sim 0 \mathrm{MeV})$. It is extremely interesting to see how microscopic calculations including the transfer of $J=0^{+}$ pairs to the $0_{g s}^{+}$states compare with the experimental data, thus improving our understanding of the origin of the enhancement factors.
In this context, it is also important to investigate the role played by neutron-proton correlations. These correlations are presently attracting peculiar interest in the field, especially making use of radioactive ion beams. As known, multinucleon transfer reactions allow the transfer of large number of nucleons, and in order to study protonneutron correlation one has to use systems where the population of the $( \pm n p)$ channels is allowed by the $Q$-value. First measurements of this kind have been very recently performed for ${ }^{92} \mathrm{Mo}+{ }^{54} \mathrm{Fe}$ [26].

The problematic issue connected with the pair correlations is of current interest in ongoing research with radioactive beams, where for example the pairing interaction is expected to be significantly modified in nuclei with extended neutron distributions [27]. The recent works on ${ }^{11} \mathrm{Li}$ induced reactions [28] provided evidence of phonon mediated pairing interaction [29]. New measurements in the near and sub-barrier regime have been recently performed in the ${ }^{16} \mathrm{O}+{ }^{208} \mathrm{~Pb}$ [30] and ${ }^{28} \mathrm{Si}+{ }^{90,94} \mathrm{Zr}$ [31], the latter performed in direct kinematics detecting target-like recoils with a recoil mass spectrometer. The newly accumulating data are also attracting a renewed interest by theorists. Models based on semiclassical theory have been used to compute transfer cross sections in light ion induced reactions [32], and calculations have been recently performed within models based on Langevin [33] or diffusion equations [34] and Time Dependent Hartree Fock theory $[35,36]$, the latter used also to compare data at above barrier energies [37].

\section{Effects due to energy loss}

The $\mathrm{Z}$ and $\mathrm{A}$ identification capability and the large detection efficiency of PRISMA allow to follow the evolution of the reaction from the quasi elastic to the deep inelastic regime. In Figs. 2 and 3 are shown the mass distributions and associated Total Kinetic Energy Loss (TKEL) spectra of representative multinucleon transfer channels for the ${ }^{90} \mathrm{Zr}+{ }^{208} \mathrm{~Pb}$ reaction. One can clearly follow the evolution pattern as function of the number of transferred nucleons. In the case of pure neutron transfers one sees a quasi-elastic peak and an increasing strength of large energy loss components when adding neutrons. When protons are involved one observes a faster growth of large TKEL components, and beyond three-proton stripping the TKEL distributions have almost similar shapes. We remind that one detects secondary fragments and that the TKEL spectra are constructed assuming binary reactions, so the shapes may be somewhat modified with respect to the (unreconstructed) primary fragments. In general, for few nucleon transfer one has a situation typical of quasielastic processes, well defined by the $Q$ values [5], while for many nucleon transfer the shape of the mass distribution is much broader (more Gaussian-like). The yields of specific proton transfer channels $(-x p \pm y n)$ are distributed over more masses and the pure proton stripping channels become less favourable as more protons are transferred, with the centroids of the mass distributions shifting to lower values. This behaviour is strongly connected with 


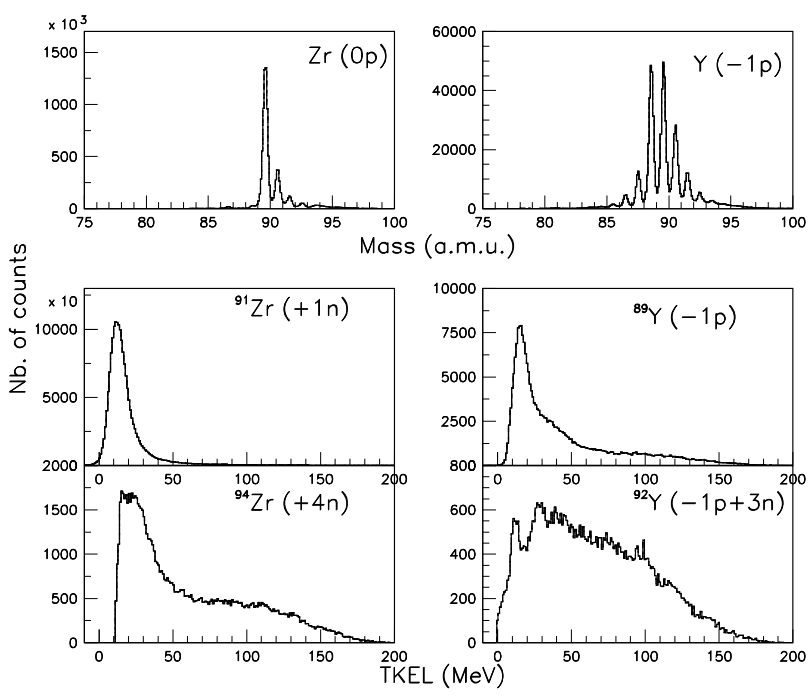

Figure 2. Mass (top panels) and TKEL (middle and bottom panels) spectra obtained in the reaction ${ }^{90} \mathrm{Zr}+{ }^{208} \mathrm{~Pb}$ at $\mathrm{E}_{\text {lab }}=560 \mathrm{MeV}$ for the indicated transfer channels. $(0 p)$ and $(-1 p)$ in the mass spectra refer to $\mathrm{Zr}$ and $\mathrm{Y}$ isotopes, respectively
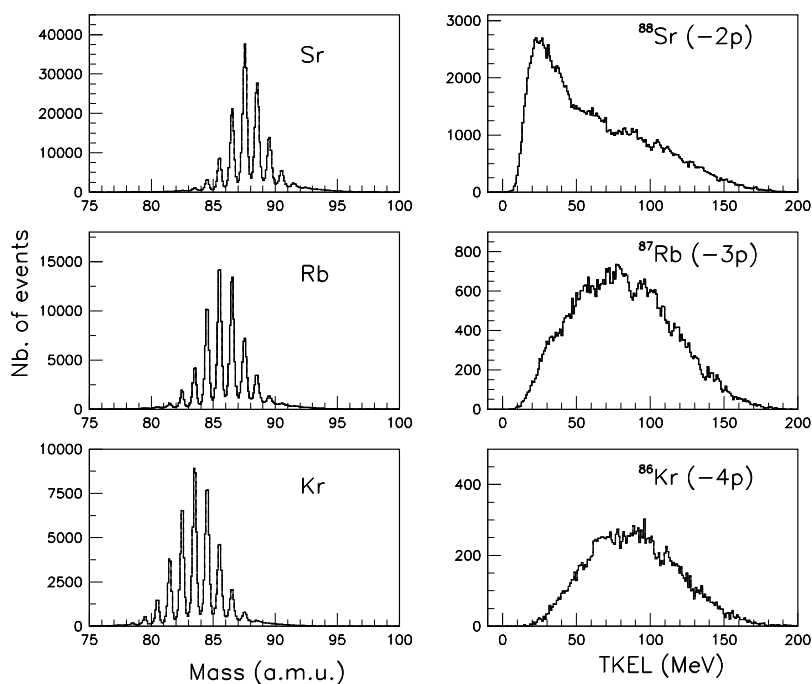

Figure 3. Mass and TKEL spectra obtained in the reaction ${ }^{90} \mathrm{Zr}+{ }^{208} \mathrm{~Pb}$ for the indicated proton stripping transfer channels

the effect of nucleon evaporation from the primary fragments, in turn associated with large energy losses [2, 3]. The importance of neutron evaporation in the modification of the final yield distribution can be directly seen through $\gamma$-particle coincidences. Gating on a specific $Z$ and $A$ (light partner) identified with a spectrometer, the velocity vector of the undetected heavy partner can be evaluated and applied for the Doppler correction of its corresponding $\gamma$ rays. In those spectra not only the $\gamma$ rays belonging to the primary binary partner are present, but also the ones of the nuclei produced after evaporation takes place. An example is given in Fig. 4 for the $(-2 p),(-2 p+1 n)$ and $(-2 p+2 n)$ channels populated in the ${ }^{40} \mathrm{Ca}+{ }^{96} \mathrm{Zr}$ reaction [10].

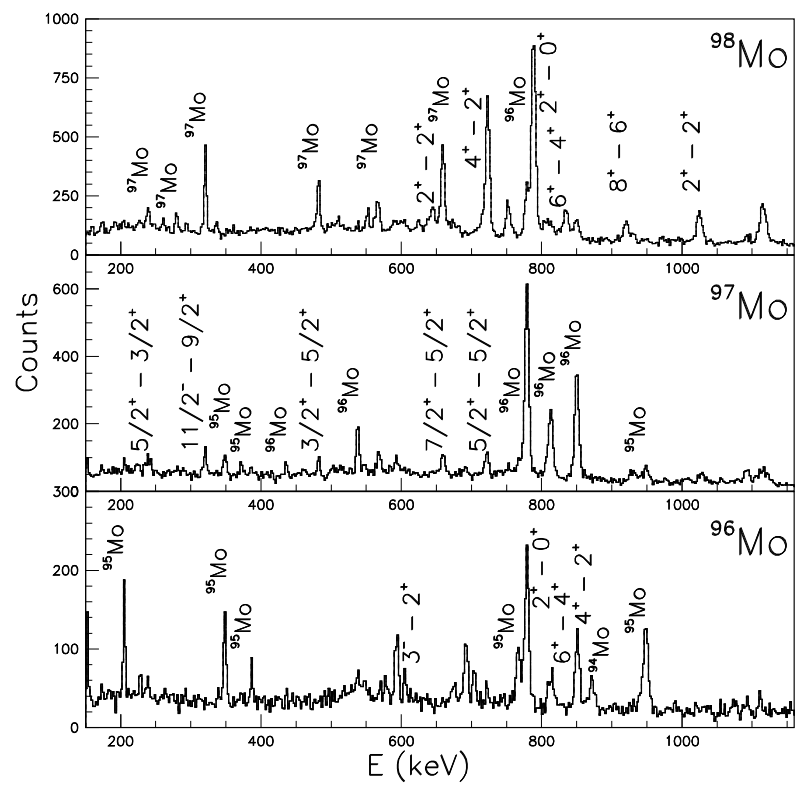

Figure 4. $\gamma$ spectra obtained in the reaction ${ }^{40} \mathrm{Ca}+{ }^{96} \mathrm{Zr}$, Doppler corrected for the heavy fragments. The main labels ${ }^{98} \mathrm{Mo},{ }^{97} \mathrm{Mo}$ and ${ }^{96} \mathrm{Mo}$ in each frame indicate the primary heavy fragments, populated via the $(-2 p),(-2 p+1 n)$ and $(-2 p+2 n)$ channels, respectively, and whose spectra correspond to ${ }^{38} \mathrm{Ar},{ }^{39} \mathrm{Ar}$ and ${ }^{40} \mathrm{Ar}$ tagged with PRISMA

\section{Population of neutron rich heavy fragments}

Besides the "light" partner products, the "heavy" partners are presently receiving peculiar attention. In fact, certain regions of the nuclear chart, like that below ${ }^{208} \mathrm{~Pb}$ or in the actinides, can be hardly accessed by fragmentation or fission reactions, and multinucleon transfer may be a suitable mechanism (if not the only one) to approach those neutron rich areas. We remind, as an example, that nuclear properties of neutron rich nuclei around $\mathrm{N}=126$ are relevant for the r-process, and play a critical role for theoretical predictions of the synthesis of the heaviest elements [33], for disentangling a variety of astrophysical scenarios [38, 39], and to study the competition between Gamow-Teller and First-Forbidden $\beta$ transitions [39].

To understand how one can approach the neutron rich heavy region, we have to keep in mind that transfer processes are governed by form factors and optimum $Q$-value considerations. With neutron poor projectiles on heavy targets only proton stripping and neutron pick-up channels are available, while with neutron rich projectiles also proton pick-up and neutron stripping channels open up 
[40]. This corresponds, for the heavy partner, to the population in the "south-east" direction, leading to the neutron rich heavy region. For the characteristic behaviour of the binding energy, the process is essentially governed by the lighter partner of the reaction [40]. This change of population pattern has been experimentally shown in different heavy ion reactions by directly detecting in $\mathrm{Z}$ and A the light partner products. In addition, as outlined before, through $\gamma$-particle coincidences, "heavy" recoils have been identified via their associated $\gamma$ rays $[10,41]$. Important new projects have been launched by different groups to produce neutron rich heavy nuclei near the $\mathrm{N}=126$ region via multinucleon transfer reactions, extract them from a gas cell and identify the products to perfom $\beta-\gamma$ coincidences $[42,43]$.

\section{Measurement of the ${ }^{197} \mathrm{Au}+{ }^{130} \mathrm{Te}$ system}

Direct detection of the heavy products $(\mathrm{A} \sim 150-200)$ is notoriously very difficult. One has to keep in mind that the bombarding energy must be close to the Coulomb barrier as a compromise between having high primary cross sections and reasonable final (detected) yield after secondary processes occur. Due to the low ion kinetic energies, A and $Z$ resolutions become worse. Significant progress can be made by using reactions in inverse kinematics, where ions are forward focused (high efficiency) and with high kinetic energy [22, 44]. Following the experience gained in

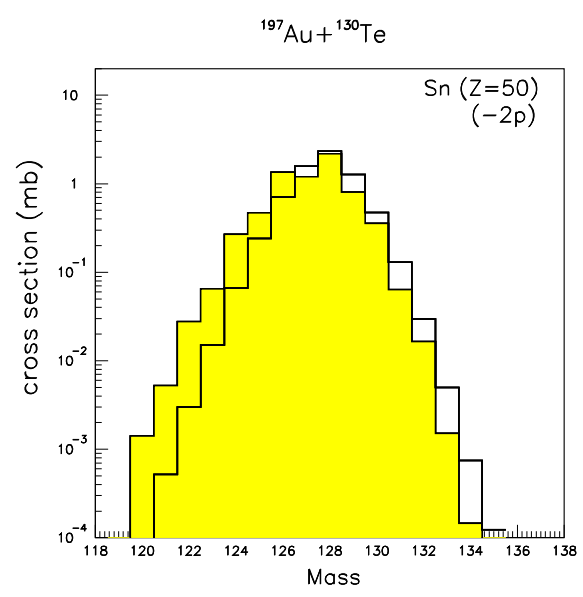

Figure 5. Theoretical cross sections for tin isotopes produced in the ${ }^{197} \mathrm{Au}+{ }^{130} \mathrm{Te}$ reaction at $\mathrm{E}_{\text {lab }}=1070 \mathrm{MeV}$. Calculations have been performed with the code GRAZING [8]. The two histograms correspond to the cases without and with neutron evaporation, the latter corresponding to lower average mass distributions.

the measurements at sub-barrier energies we recently performed an exploratory run to study the multineutron and multiproton transfer channels in the reaction ${ }^{197} \mathrm{Au}+{ }^{130} \mathrm{Te}$ at $\mathrm{E}_{l a b}=1070 \mathrm{MeV}$. The first physics goal was to get the $\mathrm{A}, \mathrm{Z}$ and Q-value distributions measuring the "light" reaction products and compare final yields with those expected from theoretical models, already successfully applied for lower mass systems [5]. In particular, via the 2 proton stripping $(-2 p)$ and 4 neutron pick-up $(+4 n)$ channels one should be able to populate ${ }^{132} \mathrm{Sn}$, a benchmark nucleus $[16,17]$. As an example, theoretical predictions [8] for the cross sections of tin isotopes are displayed in Fig. 5, from which one sees the effect of neutron evaporation. The second goal was to compare the yields of the "light" partner with those of the "heavy" one. As said before, with neutron rich projectiles proton pick-up and neutron stripping channels open up. For the heavy partner this is reflected in the population "south-east" from the injection point, leading to the neutron rich $\mathrm{Pt}-\mathrm{Os}$ heavy region. Competitive processes, i.e. evaporation and fission, may significantly shift the final yield to lower mass values and it is therefore extremely important to get experimental information on the yield distributions and compare them with theoretical predictions.

We used a 2 pnA ${ }^{197} \mathrm{Au}$ beam delivered by the PIAVE+ALPI accelerator complex of LNL onto a 100 $\mu \mathrm{g} / \mathrm{cm}^{2}{ }^{130} \mathrm{Te}$ target with a purity of $99.6 \%$. We detected with PRISMA, at an angle close to the grazing one $\left(\theta_{\text {lab }}=37^{\circ}\right.$, same at this energy for the two partners), projectile-like and target-like ions, setting different magnetic fields for the two kind of ions in two different runs.

To get the best possible ion identification, we used in-

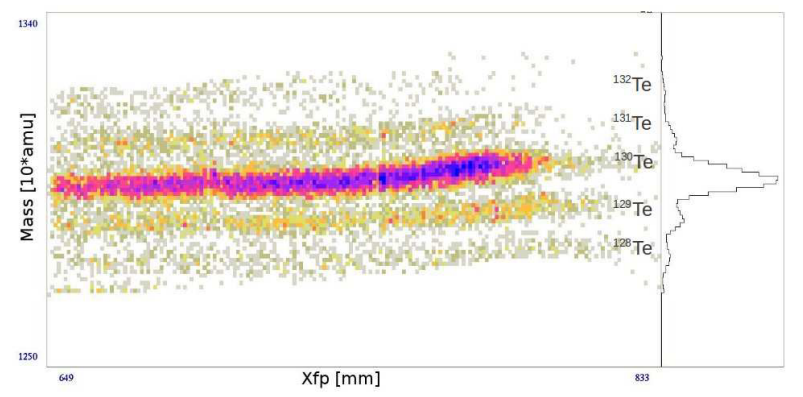

Figure 6. The matrix of mass vs position at the focal plane for Te isotopes detected in PRISMA in the ${ }^{197} \mathrm{Au}+{ }^{130} \mathrm{Te}$ reaction at $\mathrm{E}_{l a b}=1070 \mathrm{MeV}$ and $\theta_{\text {lab }}=37^{\circ}$. The different bands correspond to neutron stripping and pick-up channels. The right side shows the projection on the mass axis. Only one atomic charge state has been selected.

verse kinematics, where both binary partners have high kinetic energy at quite forward angles. Figure 6 shows as an example the matrix mass vs position of the focal plane for Te isotopes, obtained by selecting only one of the atomic charge states. At the measured angle $\theta_{l a b}=37^{\circ}$ Te-like recoils had energies of $\sim 5.5 \mathrm{MeV} / \mathrm{A}$. Mass identification is based on an event-by-event reconstruction of the ion trajectory inside the magnetic elements, using twodimensional entrance and exit positions and time-of-flight $[10,45]$. One sees the quality of separation between different masses, allowing to identify neutron stripping as well as neutron pick-up channels. For proton transfer channels the cross sections are roughly an order of magnitude less than the neutron transfer ones. 


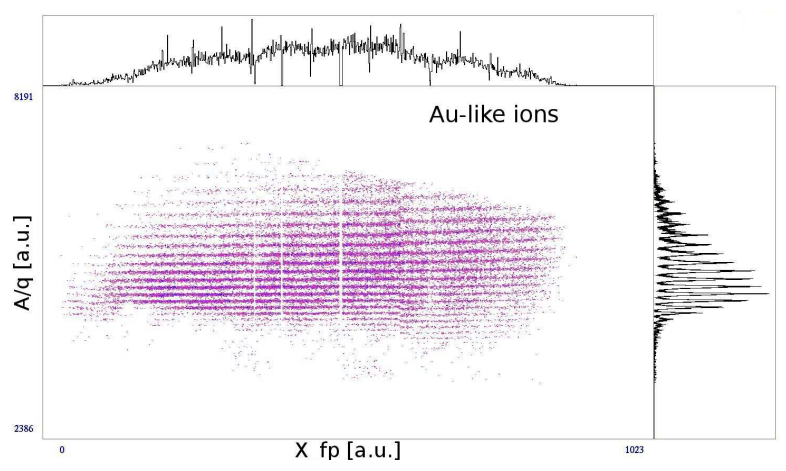

Figure 7. The matrix of mass over atomic charge state $(A / q)$ vs position at the focal plane for Au-like particles detected in the ${ }^{197} \mathrm{Au}+{ }^{130} \mathrm{Te}$ reaction at $\mathrm{E}_{\text {lab }}=1070 \mathrm{MeV}$ and $\theta_{\text {lab }}=37^{\circ}$. The projections on the two axis are also displayed. The asymmetric $A / q$ peak distribution is due to the distortion of the ion optics.

For Au-like ions, detected obviously with different magnetic fields, we got a very good $A / q$ resolution, shown in Fig. 7. The direct detection of Au-like ions was also an excellent opportunity to test for the first time the detector performance of PRISMA with large masses (actually with the highest beam mass presently available at $\mathrm{LNL}$ ), a test important and necessary for future studies and developments. Work is underway to improve the tracking algorithm, necessary to properly extract the atomic charge state distributions of these very heavy ions. In the next period a signifcant improvement of the experiment will come with the installation on the PRISMA scattering chamber of a second time of flight system to perfom high resolution kinematic coincidences. With the additional information provided by this second arm, equipped with position sensitive microchannel-plate, parallel-plate and Bragg ionization chamber, we will be able to associate the mass and nuclear charge distributions of heavy transfer products with each isotope identified in PRISMA. We expect to get important information on the production yield of heavy neutron rich partners and to measure the probability of transfer induced fission (see next paragraph).

\section{Fission products}

As a further exploratory run, we tested the reaction ${ }^{197} \mathrm{Au}+{ }^{130} \mathrm{Te}$ at at $\mathrm{E}_{l a b}=1300 \mathrm{MeV}$ and at a more forward angle $\theta_{\text {lab }}=27^{\circ}$, setting the magnetic fields and the gas detectors to directly measure "fission-like" products. The corresponding energy-range matrix is displayed in Fig. 8. One can observe, besides the bulk of Au-like ions, two main distributions, presumably coming from the near symmetric fission of Au (lower branch) and the convolution of quasi-fission, transfer-induced fission and deepinelastic components (upper branch). Of course, lacking a true kinematic coincidence we could not quantitatively attribute those event distributions to specific reaction mechanisms, which could overlap in parts of their angular and Q-value distributions selected by the spectrometer. On the other hand we could assess the feasibility of these studies.
Indeed, the individual bands, associated with the different nuclear charges, show the obtained $\mathrm{Z}$ resolution near the $\mathrm{Z} \sim 40-50$ region, quite similar to what was recently performed using the VAMOS spectrometer in GANIL with the specific purpose to study the yields distributions of fission products [46].

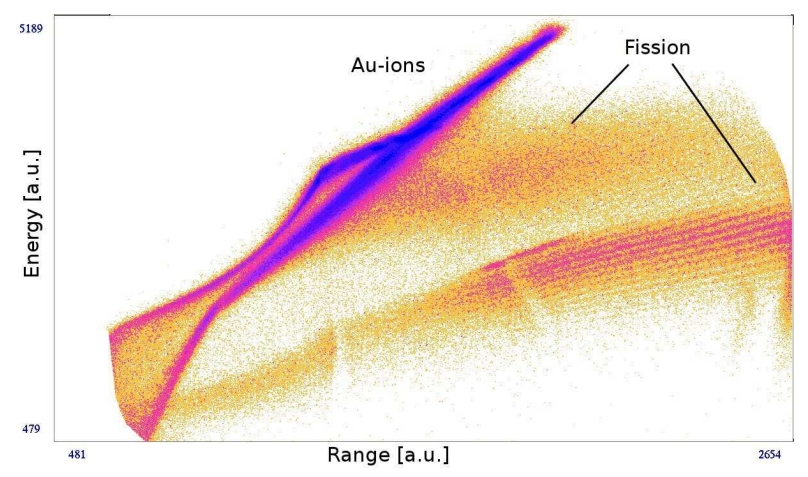

Figure 8. Energy vs range (in the ionization chamber) matrix obtained in the reaction ${ }^{197} \mathrm{Au}+{ }^{130} \mathrm{Te}$ at at $\mathrm{E}_{\text {lab }}=1300 \mathrm{MeV}$ and $\theta_{\text {lab }}=27^{\circ}$. The magnetic fields and gas detector system of PRISMA have been set to measure "fission-like" fragments (see text for details).

The mechanism of fission deserves some comments, in particular transfer induced fission, affecting generally the heavy partner. From the point of view of $\gamma$ spectroscopy, this turns out to be an efficient way to populate neutron rich nuclei. First experiments where fission products are selected in $\mathrm{A}$ and $\mathrm{Z}$ with a spectrometer and their associated $\gamma$ rays are detected with large $\gamma$ arrays have been already performed. For instance, the reaction ${ }^{136} \mathrm{Xe}+{ }^{238} \mathrm{U}$ [47] has been employed to measure the lowest energy levels of ${ }^{96} \mathrm{Kr}$ and study the evolution of quadrupole deformation in that region. The ${ }^{238} \mathrm{U}+{ }^{12} \mathrm{C}$ reaction [48] has been used to populate neutron rich nuclei in the mass range $\mathrm{A} \sim 100-140$, where properties of newly identified levels have been compared with large scale shell model calculations.

As said before, the competitive processes of evaporation and/or fission shift the final yield to lower mass values. Fission becomes of course more and more crucial in governing the decay process in the actinides and transactinides region [49]. It is therefore extremely important to get quantitative information on the final yield distributions and compare them with theoretical predictions. High resolution kinematic coincidences are expected to bring important information on these processes. As an example, in the ${ }^{58} \mathrm{Ni}+{ }^{208} \mathrm{~Pb}$ measurement of Ref. [50] the relevance of transfer induced fission and how the experimental values can be quite well described by the same theory used to compare with the distributions of the light partner have been discussed.

\section{Conclusions}

Significant advances have been made in the last years in the field of multinucleon transfer reactions. With mul- 
tiple transfers of neutrons and protons one could populate nuclei moderately far from stability, especially in the neutron-rich region, important for both reaction mechanism and nuclear structure. Valuable information on nucleon-nucleon correlations could be also derived, particularly in studies below the Coulomb barrier. Present focus is also in the study of the properties of the heavy binary partner, important for astrophysics. The presence of secondary effects, namely nucleon evaporation and transfer induced fission, need to be more carefully investigated, especially near the $\mathrm{Pb}$ and in the actinide regions, where other production methods, like fission or fragmentation, have severe limitations.

\section{Acknowledgements}

The material presented in this paper is the result of the work of many people which I would like to thank, in particular S. Szilner, T. Mijatović, N. Soić, D. Jelavić Malenica (RBI, Croatia), G. Pollarolo (TO, Italy), E. Fioretto, A. M. Stefanini, J. J. Valiente-Dobón (LNL, Italy), D. Montanari, G. Montagnoli, F. Scarlassara, C. Michelagnoli, C. A. Ur (PD, Italy), S. Courtin, A. Goasduff, F. Haas (Strasbourg, France), D. Ackermann (GSI, Germany), Y. Watanabe, S. Jeong (KEK, Japan)

\section{References}

[1] R. A. Broglia and A. Winther Heavy Ion Reactions (Addison-Wesley Pub. Co., Redwood City CA, 1991)

[2] L. Corradi et al., Phys. Rev. C 59, 261 (1999)

[3] S. Szilner et al., Phys. Rev. C 71, 044610 (2005)

[4] G. Pollarolo, Phys. Rev. Lett. 100, 252701 (2008)

[5] L. Corradi, G. Pollarolo and S. Szilner, J. of Phys. G: Nucl. Part. Phys. 36, 113101 (2009)

[6] A. Winther, Nucl. Phys. A 572, 191 (1994)

[7] A. Winther, Nucl. Phys. A 594, 203 (1995)

[8] A. Winther, program GRAZING, http:/www.to.infn.it/ nanni/grazing

[9] A. M. Stefanini et al., Nucl. Phys. A 701, 217c (2002)

[10] S. Szilner et al., Phys. Rev. C 76, 024604 (2007)

[11] H. Savajols et al., Nucl. Phys. A 654, 1027c (1999)

[12] A. Cunsolo et al., Nucl. Instr. and Meth. in Phys. Res. A 481, 48 (2002)

[13] A. Gadea et al., Eur. Phys. J. A 20, 193 (2004)

[14] S. L. Shepherd et al., Nucl. Instr. and Meth. in Phys. Res. A 434, 373 (1999)

[15] A. Gadea et al., Nucl. Instr. and Meth. in Phys. Res. A 654, 88 (2011)

[16] http://www.ganil.fr/research/developments/spiral2 The Scientific Objectives of the Spiral2 Project (2006)

[17] SPES: (http://web.infn.it/spes/) Exotic beams for science
[18] L. Corradi et al., Nucl. Instr. and Meth. B 317, 743 (2013)

[19] B. F. Bayman and J. Chen, Phys. Rev. C 26, 1509 (1982)

[20] E. Maglione, G. Pollarolo, A. Vitturi, R. A. Broglia and A. Winther, Phys. Lett. B 162, 59 (1985)

[21] J. H. Sorensen and A. Winther, Nucl. Phys. A 550, 306 (1992)

[22] L. Corradi et al., Phys. Rev. C 84, 034603 (2011)

[23] W. von Oertzen et al., Eur. Phys. J. A 20, 153 (2004)

[24] D. Montanari et al., Phys. Rev. Lett. 113, 052501 (2014)

[25] S. Szilner et al., Eur. Phys. J. A 21, 87 (2004)

[26] S. Szilner et al., contribution to this conference

[27] J. Dobaczewski, I. Hamamoto, W. Nazarewicz, and J. A. Sheikh, Phys. Rev. Lett. 72, 981 (1994)

[28] I. Tanihata et al., Phys. Rev. Lett. 100, 192502 (2008)

[29] G. Potel, F. Barranco, E. Vigezzi and R. A. Broglia, Phys. Rev. Lett. 105, 172502 (2010)

[30] M. Evers et al., Phys. Rev. C 84, 054614 (2011)

[31] S. Kalkal et al., Phys. Rev. C 83, 054607 (2011)

[32] G. Potel et al., Rep. Prog. Phys. 76, 106301 (2013)

[33] V. Zagrebaev and W. Greiner, Phys. Rev. Lett. 101, 122701 (2008)

[34] V.V. Sargsyan et al., Phys. Rev. C 88, 064601 (2013)

[35] C. Simenel, Phys. Rev. Lett. 105, 192701 (2010)

[36] G. Scamps and D. Lacroix, Phys. Rev. C 87, 014605 (2013)

[37] K. Sezikawa and K. Yabana, Phys. Rev. C 88, 014614 (2013)

[38] K.-L. Kratz et al., Astrophys. J. 403, 216 (1994)

[39] H. Grawe et al., Rep. Prog. Phys. 70, 1525 (2007)

[40] C. H. Dasso, G. Pollarolo and A. Winther, Phys. Rev. Lett. 73, 1907 (1994)

[41] Y. Watanabe et al., Nucl. Instr. and Meth. B 317, 752 (2013) and contribution to this conference

[42] S. C. Jeong, Construction proposal for KEK Isotope Separation System for $\beta$-decay spectroscopy, RIBF NPPAC-06, 2009.

[43] S. Zemlyanoy, contribution to this conference

[44] C. L. Jiang et al., Phys. Rev. C 57, 2393 (1998)

[45] D. Montanari et al., Eur. Phys. J. A 47, 4 (2011)

[46] M. Caamano et al., Phys. Rev. C 88, 024605 (2013)

[47] N. Marginean et al., Phys. Rev. C 80, 021301(R) (2009)

[48] A. Shrivastava et al., Phys. Rev. C 80, 051305R (2009)

[49] V.I. Zagrebaev and W. Greiner, Phys. Rev. C 87, 034608 (2013)

[50] L. Corradi et al., Phys. Rev. C 66, 024606 (2002) 